\title{
On the origin of irregular structure in Saturn's rings
}

\author{
Scott Tremaine \\ Princeton University Observatory, Peyton Hall, Princeton, NJ 08544 \\ tremaine@astro.princeton.edu
}

\begin{abstract}
We suggest that the irregular structure in Saturn's B ring arises from the formation of shear-free ring-particle assemblies of up to $\sim 100 \mathrm{~km}$ in radial extent. The characteristic scale of the irregular structure is set by the competition between tidal forces and the yield stress of these assemblies; the required tensile strength of $\sim 10^{5} \mathrm{dyn} \mathrm{cm}^{-2}$ is consistent with the sticking forces observed in laboratory simulations of frosted ice particles. These assemblies could be the nonlinear outcome of a linear instability that occurs in a rotating fluid disk in which the shear stress is a decreasing function of the shear. We show that a simple model of an incompressible, non-Newtonian fluid in shear flow leads to the Cahn-Hilliard equation, which is widely used to model the formation of structure in binary alloys and other systems.
\end{abstract}

Subject headings: planets: rings — celestial mechanics

\section{Introduction}

The Voyager 1 and 2 spacecraft, which flew past Saturn in 1980 and 1981, revolutionized our understanding of the Saturn system. One of the remarkable features discovered by Voyager was rich radial structure in Saturn's rings. Although some of this structure, mostly in the outer or A ring, is known to arise from density or bending waves generated by discrete resonances with the inner satellites, most of the radial structure remains unexplained, particularly in the main or B ring. Horn \& Cuzzi (1996) point out that wavetrains associated with known resonances cover less than $1 \%$ of the radial extent of the $\mathrm{A}$ and $\mathrm{B}$ rings. The remaining vast majority of the structure is often called "irregular" since the bright and dark features show little or no long-range coherence.

The purpose of this paper is to suggest and examine a novel explanation for irregular structure in Saturn's rings. Ring particles are likely to have (weak) cohesive forces, and 
therefore can assemble or "freeze" into structures much larger than an individual ring particle. The size of these structures is limited by tidal forces from Saturn and collisional erosion by impacting particles. We suggest that much of the irregular structure may consist of alternating annuli of "solid" and "liquid" ring material, the former consisting of an assembly of ring particles frozen into rigid rotation around Saturn, and the latter consisting of individual ring particles in differential rotation. Thus we hypothesize that the irregular structure is primarily a manifestation of variations in shear rather than surface density.

Rich spatial structure associated with the co-existence of two phases is a feature of many physical processes, including ferromagnetism, spinodal decomposition in alloys, crystal growth, chemical reactions, and even traffic flow. We shall exploit some of these analogies in $§ 5$. One complication in Saturn's rings is that the physics is non-local, because the tidal stresses on a rigidly rotating annulus depend on its total radial extent. We estimate these stresses and the corresponding upper limit to the radial extent of rigidly rotating annuli in $\S 4.2$.

We begin by reviewing the observations of the irregular structure in $\S 2$ and competing theoretical explanations in $\S 3$. In $\S 4$ we review the equations that describe the dynamics of the ring fluid. In $\S 5$ we describe a simple toy model that illustrates some of the important dynamical behavior associated with the irregular structure, and sets the model in the context of the theory of phase transitions.

The suggestion that some areas in planetary rings are locked into solid assemblies is not new to this paper. Wisdom \& Tremaine (1988) point out that dense rings may have a liquidsolid phase transition; they did not find any such transition in their numerical experiments on inelastically colliding hard spheres, but this is not surprising since they did not model any cohesive forces. Mosqueira \& Estrada (2002) suggest that ring-particle shocks or "jams" may occur in sectors of converging flow in an eccentric ring; however, our focus is on axisymmetric ring-particle structures held together by cohesive forces, while theirs is on non-axisymmetric structures held together by self-gravity and ram pressure.

\section{Observations}

Voyager images reveal irregular structure throughout the $\mathrm{A}$ and $\mathrm{B}$ rings, over a wide range of scales ranging from several hundred $\mathrm{km}$ to the resolution limit of about $5 \mathrm{~km}$. In regions where the ring is sufficiently transparent, the surface-brightness variations track the optical-depth variations detected during stellar occultations observed by Voyager (Horn \&

Cuzzi 1996). However, in the main part of the B ring the optical depth is so high that there 
is little or no signal from the occultation experiments; moreover, the brightness variations in this region cannot be explained by optical depth variations, since the solar elevation at the time of the encounter was so low $\left(\lesssim 8^{\circ}\right)$ that variations in optical depth have negligible influence on the surface brightness in reflected light. Thus, other effects such as particle albedo or phase function, which may or may not be correlated with optical depth variations, must be responsible for most of the irregular structure in the B ring. Comparison of images at different phase angles (Cuzzi et al. 1984) suggests that some features are due to variations in albedo and others due to variations in phase function. The surface-brightness ratios at different phase angles appear to be bimodal, suggesting that there are only two distinct phase functions.

Most of the irregular structure appears to be axisymmetric, at least on scales $\gtrsim 50 \mathrm{~km}$ and away from major resonances. At smaller scales the features at the same radius and different longitudes cannot be matched, implying that they are non-axisymmetric or timevariable or both.

Horn \& Cuzzi (1996) have measured the local power spectrum of the reflectivity of the $\mathrm{B}$ ring as a function of radius (from 92,000 km at its inner edge to 122,000 km at the Cassini division). In most regions the dominant wavelength varies between 100 and $200 \mathrm{~km}$. In the outer $1500 \mathrm{~km}$ of the ring, and a few other isolated regions, there is substantial power at wavelengths as short as tens of $\mathrm{km}$. There are also regions of $1000 \mathrm{~km}$ or so in which little or no irregular structure is visible.

The particle-size distribution in Saturn's rings is constrained by the Voyager occultation experiments (Zebker, Marouf, \& Tyler 1985; Showalter \& Nicholson 1990). These observations suggest a broad distribution of particle sizes with an upper cutoff of a few meters, but cannot distinguish whether these particles are in differential rotation or locked by contact forces into a solid assembly.

\section{Theoretical models}

The irregular structure is surprising because viscous diffusion is expected to smooth out such structure on timescales much less than the age of the rings. The characteristic time required to smooth out structure on a radial scale $\Delta r$ is

$$
t_{\nu}=\frac{(\Delta r)^{2}}{\nu}=3 \times 10^{4} \mathrm{y}\left(\frac{\Delta r}{100 \mathrm{~km}}\right)^{2}\left(\frac{100 \mathrm{~cm}^{2} \mathrm{sec}^{-1}}{\nu}\right),
$$

where $\nu$ is the kinematic viscosity, which can be estimated either from the rate of damping of density waves, or from kinetic theory together with estimates of the particle size and 
optical depth. The very small value of $t_{\nu}$ implies that all small-scale radial structure should be erased from the $\mathrm{B}$ ring, unless it is actively maintained by some mechanism.

One possible explanation is that the irregular structure arises from gravitational shepherding by small moonlets or large particles. However, objects large enough to influence the ring over scales $\gtrsim 100 \mathrm{~km}$ should also clear a gap around themselves, and such gaps are not seen (Esposito, O'Callaghan, \& West 1983).

One attractive hypothesis is that the irregular structure arises from local axisymmetric instabilities in the rings. There are two natural candidates for this instability:

- Viscous instability. In most models for axisymmetric ring dynamics, the kinematic viscosity $\nu$ is assumed to be a function only of the surface density $\mu$. The viscous instability then arises if the angular-momentum flux in the rings is a decreasing function of surface density, that is, if

$$
K_{1} \equiv-\frac{d(\mu \nu)}{d \mu}>0
$$

the growth rate of the instability is $\gamma=3 k^{2} K_{1}$, where $k$ is the radial wavenumber. This is a secular instability, since the growth rate is proportional to the strength of the dissipative forces (i.e., $\gamma \propto \nu$ ). If condition (2) is satisfied, ring material preferentially migrates from regions of low surface density to regions of high surface density, thereby adding mass to the high-density regions, and depleting the low-density regions even further.

The viscous instability was first discussed in the context of accretion disks by Lightman \& Eardley (1974), and was invoked to explain the irregular structure in Saturn's rings by Lin \& Bodenheimer (1981), Lukkari (1981), and Ward (1981). These authors were motivated by the observation that the viscosity in dilute rings appears to satisfy the instability condition (2). However, the B ring is unlikely to be dilute, unless the elasticity of its constituent particles is unrealistically high. Both analytic kinetic theory and $N$-body simulations of the viscosity in a dense ring composed of inelastic hard spheres show that the instability condition (2) is not satisfied by a wide margin (Araki \& Tremaine 1986; Wisdom \& Tremaine 1988); therefore, the viscous instability is unlikely to operate in Saturn's B ring.

- Viscous overstability. Density waves can propagate in planetary rings, and dozens of density wavetrains have been identified in Saturn's A ring. The collective effect that dominates density-wave propagation is the self-gravity of the ring. Self-gravity is important, even though the ring is much less massive than the planet, because the thickness of the ring is also much less than its radius. A more precise statement is that 
Toomre's $Q$ parameter is of order unity in the rings. Viscosity can lead to either decay or growth of density waves; the latter case, known as viscous overstability, occurs if

$$
K_{2} \equiv 3 \mu \frac{d \nu}{d \mu}+\frac{2}{3} \nu-\xi>0
$$

here $\nu$ and $\xi$ are the kinematic shear and bulk viscosity, and both are assumed to depend only on the surface density $\mu$. The growth rate is $\gamma=\frac{1}{2} k^{2} K_{2}$. Unfortunately, the assumptions that the stress tensor can be represented by a shear and bulk viscosity, and that these depend only on the surface density, are harder to justify than in the case of the viscous instability, since an overstable ring oscillates on a timescale comparable to the interparticle collision time.

The existence of this instability was first pointed out by Kato (1978) for accretion disks (where the important collective effect is pressure rather than self-gravity), and by Goldreich \& Tremaine (1978) for planetary rings. Borderies, Goldreich \& Tremaine (1985) describe a crude kinetic theory for a ring composed of inelastic, closely packed, hard spheres, and show that the viscous instability is present in this approximation. Schmidt \& Tscharnuter (1995) discuss in detail the local linear stability of a differentially rotating, two-dimensional, isothermal, fluid disk, in which the viscosity depends only on surface density, and the effects of self-gravity are included; they derive a cubic dispersion relation that contains the viscous instability and viscous overstability as special cases. Schmidt \& Tscharnuter (1999) describe numerical calculations of the nonlinear evolution of these disks, which show that the viscous overstability can lead to a rich radial structure with surface-density contrast of order unity. Numerical experiments on self-gravitating collections of ring particles appear to exhibit a viscous overstability in some cases (Mosqueira 1996; Daisaka, Tanaka, \& Ida 2001; Salo, Schmidt \& Spahn 2001). However, the most unstable wavelengths in the linear calculations, and the wavelengths with significant power in the nonlinear calculations, are at most a few hundred meters - almost three orders of magnitude smaller than the dominant wavelengths in the irregular structure in the B ring (Horn \& Cuzzi 1996). Schmidt \& Tscharnuter (1999) argue that nonlinear wave-wave interactions transfer the fluctuation power to much larger wavelengths, but so far this suggestion remains untested.

A quite different proposal is that some of the irregular structure arises from unstable ballistic transport (Lissauer 1984; Durisen et al. 1989, 1992; Durisen 1995). Impacts by interplanetary particles erode the ring particles and redistribute their mass to adjacent annuli. This process is unstable because high-density regions of the ring tend to absorb more of the ejecta than neighboring regions. However, the growth rate of the instability is negligible for 
optical depths $\gtrsim 1.5$. Thus, ballistic transport is probably unable to explain the irregular structure in the bulk of the $\mathrm{B}$ ring.

\section{Shear stress in dense planetary rings}

In this section we investigate the dynamics, stresses, and stability of solid and liquid ring phases.

\subsection{Equations of motion}

We examine a ring orbiting a point mass $M$. Test particles on circular orbits travel at the Keplerian angular speed $\Omega_{K}(r)=\left(G M / r^{3}\right)^{1 / 2}$. We employ a rotating Cartesian coordinate system in which $\hat{\mathbf{e}}_{x}$ points radially outward, $\hat{\mathbf{e}}_{y}$ points in the direction of rotation, and the origin, at radius $R$, rotates around the central mass at angular speed $\Omega \equiv \Omega_{K}(R)$. We work at distances from the origin that are small compared to the orbital radius $R$ (Hill's approximation). We ignore the vertical structure of the disk, treating it as a razor-thin sheet with surface density $\mu(\mathbf{x}, t)$. We restrict ourselves to axisymmetric disturbances, so that $\partial / \partial y=0$. The Euler and continuity equations then read

$$
\begin{aligned}
\frac{\partial u}{\partial t}+u \frac{\partial u}{\partial x} & =3 \Omega^{2} x+2 \Omega v+\frac{1}{\mu} \frac{\partial \Sigma_{x x}}{\partial x}, \\
\frac{\partial v}{\partial t}+u \frac{\partial v}{\partial x} & =-2 \Omega u+\frac{1}{\mu} \frac{\partial \Sigma_{x y}}{\partial x}, \\
\frac{\partial \mu}{\partial t}+\frac{\partial}{\partial x}(\mu u) & =0,
\end{aligned}
$$

where $\mathbf{v}(\mathbf{x}, t)=u(x, t) \hat{\mathbf{e}}_{x}+v(x, t) \hat{\mathbf{e}}_{y}$ is the velocity in the rotating frame, and $\Sigma_{i k}=\int \sigma_{i k} d z$ is the vertically integrated stress tensor (dimensions of force per unit length). We define the tangential shear to be

$$
s \equiv \frac{\partial v}{\partial x} .
$$

In a disk with zero stress gradients, a solution of the equations of motion is

$$
u(x, t)=0, \quad v(x, t)=v_{K}(x)=-\frac{3}{2} \Omega x,
$$

corresponding to circular Keplerian orbits and constant shear $s=s_{K} \equiv-\frac{3}{2} \Omega$. In a solid disk with zero shear, a solution of the equations of motion is

$$
u(x, t)=0, \quad v(x, t)=v_{s}=\text { constant }, \quad \Sigma_{x y}=\text { constant }, \quad \frac{\partial \Sigma_{x x}}{\partial x}=-\mu\left(2 \Omega v_{s}+3 \Omega^{2} x\right) .
$$


If we assume that the surface density is constant, that the zero-shear region extends from $x_{1}$ to $x_{2}$, and that the tensile stress vanishes at the edges of the solid region (i.e. $\Sigma_{x y}=0$ at $\left.x_{1}, x_{2}\right)$ then the last of these equations can be integrated to yield

$$
\Sigma_{x x}=\frac{3}{2} \mu \Omega^{2}\left(x-x_{1}\right)\left(x_{2}-x\right), \quad v_{s}=-\frac{3}{4} \Omega\left(x_{1}+x_{2}\right) .
$$

Thus, any solid annulus is subject to a tensile stress, the maximum of which occurs at its midline and is equal to

$$
\Sigma_{x x, \max }=\frac{3}{8} \mu \Omega^{2}(\Delta x)^{2},
$$

where $\Delta x \equiv x_{2}-x_{1}$ is the width of the annulus.

If we linearize the first two of equations (4) with respect to a state of uniform shear $\left(u_{0}=0, \partial v_{0} / \partial x=s\right)$, and neglect perturbations in the stress tensor, then small disturbances are stable if and only if $s>-2 \Omega$. This is the well-known Rayleigh criterion for the stability of Couette flow (Chandrasekhar 1961).

\subsection{Tensile stress}

Equation (9) for the maximum height-integrated tensile stress (force per unit length) in a solid annulus can be converted to an equation for the ordinary tensile stress (force per unit area) by approximating the vertical structure of the ring as that of a homogeneous slab with thickness $h$ and density $\rho=\mu / h$ (note that $\rho$ is less than the density of the ring particles, by the filling factor):

$\sigma_{x x, \text { max }}=\frac{\Sigma_{x x, \max }}{h}=\frac{3}{8} \rho \Omega^{2}(\Delta r)^{2}=4 \times 10^{5} \mathrm{dyn} \mathrm{cm}^{-2}\left(\frac{\rho}{0.3 \mathrm{~g} \mathrm{~cm}^{-3}}\right)\left(\frac{10^{10} \mathrm{~cm}}{r}\right)^{3}\left(\frac{\Delta r}{100 \mathrm{~km}}\right)^{2}$,

in which we have inserted parameters appropriate for Saturn. The maximum width of a solid annulus with a given tensile strength may be called its "tidal width". If we identify the tidal width with half of the dominant wavelength of $100 \mathrm{~km}$ seen in the irregular structure (i.e. $\Delta r=50 \mathrm{~km}$ ) then we require that a frozen assembly of ring particles have a tensile strength

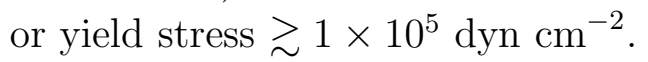

The strength of ring-particle assemblies is, of course, very difficult to estimate. An upper limit is the yield stress of solid ice, which is $\sigma_{\max } \sim 10^{7} \mathrm{dyn}^{-2}$ at temperatures 5-10 K below freezing (Schulson 1999), and probably higher at the much lower temperatures characteristic of Saturn's rings. However, the actual strength of ice-particle assemblies can be many orders of magnitude lower: comets, which may be rubble piles of icy particles, have yield stresses $\sigma_{\max } \lesssim 10^{3}$ dyn $\mathrm{cm}^{-2}$, and Asphaug \& Benz (1996) derive an even lower strength $\lesssim 10^{2}$ dyn $\mathrm{cm}^{-2}$ for Comet Shoemaker-Levy 9 . 
Experimental work on sticking of cm-sized ice particles is reviewed by Bridges et al. (1996). The experiments show no sticking unless the ice surfaces are coated with uncompacted frost; however, thin water-frost layers can lead to sticking forces up to $10^{4}$ dyn over contact areas $\sim 0.01 \mathrm{~cm}^{2}$ (see also Supulver et al. 1997). On Saturn's ring particles, frost is likely to be produced by dust-particle impacts, and removed by collisions with other particles; Hatzes et al. (1991) conclude that centimeter-sized or larger particles will be frost-coated, although this conclusion relies on a very uncertain estimate of the optical depth in grains. Whether or not frost layers are present on B-ring particles is therefore an open question; we shall assume that frost is present in order to have significant sticking forces.

If the sticking force scales with the contact area, then the forces found by Bridges et al. (1996) would imply a tensile strength per unit area of $10^{6} \mathrm{dyn}_{\mathrm{cm}}^{-2}$. This is certainly an overestimate, since the contact area between ring particles is only a fraction of the total area. If there is a wide range of particle sizes, the contact points and the yield stress are likely to be dominated by small particles, which provide a kind of "cement" to hold the large particles together. We now make a crude estimate of the yield stress. For spherical particles of radius $R$, the contact area is $A=2 \pi R d$ where $d$ is the thickness of the frost layer. We assume that the contact force is proportional to the contact area, that the force is $\approx 10^{4}$ dyn for a contact area $A=0.01 \mathrm{~cm}^{2}$, that the thickness of the frost layer is $d \approx 10^{-2} \mathrm{~cm}$, and that there are $N \approx 1 \mathrm{~cm}^{2} / \pi R^{2}$ contacts per $\mathrm{cm}^{2}$. Then the yield stress is $\sigma_{\max } \approx 2 \times 10^{4}(1 \mathrm{~cm} / R) \mathrm{dyn}^{-2}$, comparable to the required yield stress if $R \approx 0.2 \mathrm{~cm}$. Note that most of the mass of the ring could be - and likely is - in much larger particles, while most of the sticking force comes from the small particles.

Therefore, within the very large uncertainties, it is plausible that annular ring-particle assemblies as large as $\Delta r \sim 50-100 \mathrm{~km}$ could have the required strength $\left(\sigma_{\max } \sim 10^{5} \mathrm{dyn}_{\mathrm{cm}}^{-2}\right)$ to survive tidal stresses.

We now briefly discuss the orbital stability of solid annuli. Laplace and later Maxwell showed that a solid annulus orbiting a planet is unstable, with growth time $\Omega / \sqrt{ } 2$. However, Fridman, Morozov, \& Polyachenko (1984) point out that the Laplace-Maxwell analysis assumes that the annulus is completely rigid, in particular that the internal sound speed $c \gg \Omega r$. In fact $c \ll \Omega r \simeq 20 \mathrm{~km} \mathrm{~s}^{-1}$ for any plausible material. In this case the nature of the instability changes dramatically (Fridman, Morozov, \& Polyachenko 1984): the growth rate becomes much smaller, $\sqrt{3} m c / r$, where $m$ is the azimuthal wavenumber; the instability is predominantly azimuthal; and the $m=1$ instability in the rigid ring is replaced by an instability that is present for all $m$ and grows faster as $m$ increases. The physical basis for the instability is simple: if a mass element moves closer to the element in front of it, it is pushed backwards by elastic forces. Thus it loses angular momentum, its angular speed 
increases, and it moves even closer to the element in front.

One limitation of the analysis of Fridman, Morozov, \& Polyachenko (1984) is the neglect of the rigidity of the ring: they assume that the modulus of compression is non-zero but the modulus of rigidity $\mu$ is zero. Rigidity suppresses the instability at short wavelengths, for $m>m_{\text {crit }} \equiv(3 \rho / \mu)^{1 / 2} \Omega r$, where $\rho$ is the density in the ring.

Thus there are several possibilities that may enable solid ring-particle assemblies to be stable. Possibly the rigidity is large enough to stabilize all azimuthal wavenumbers; possibly the solid regions are arcs, rather than annuli, spanning angles $\lesssim 2 \pi / m_{\text {crit }}$; possibly there is a slowly growing instability that disrupts the solid annuli, so that they are constantly dissolving and re-forming; or perhaps other effects such as pressure from adjacent parts of the ring can stabilize a solid annulus. We cannot say which of these possibilities is more likely, but it would be premature to dismiss the possibility of solid annuli in the ring on the basis of stability arguments.

\subsection{Shear stress}

The simplest model for the shear stress is $\Sigma_{x y}=E s$, where $E=\int \eta d z$, the vertically integrated viscosity, is assumed to be a function only of the surface density $\mu$. Many authors have investigated the stability and evolution of models of this kind (Lightman \& Eardley 1974; Goldreich \& Tremaine 1978; Kato 1978; Lin \& Bodenheimer 1981; Schmidt \& Tscharnuter 1995, 1999). However, granular systems generally behave as non-Newtonian fluids, in which the viscosity is a function of the shear, so that the shear stress $\Sigma_{x y}$ is likely to be a nonlinear function of the shear.

For example, a simple kinetic theory for dense planetary rings is described by Borderies, Goldreich \& Tremaine (1985). In their model, the shear stress and radial stress are

$$
\Sigma_{x y}=q_{1} F \frac{\mu^{3} \Omega^{2}}{\rho^{2}} \operatorname{sgn}(s), \quad \Sigma_{x x}=-q_{2} F \frac{\mu^{3} \Omega^{2}}{\rho^{2}} ;
$$

here $q_{1}$ and $q_{2}$ are dimensionless constants of order unity, and $\rho$ is the density of the ring particles or the mean density inside the ring (the theory is not accurate enough to distinguish these two densities, as it assumes in effect that the ring is incompressible). The dimensionless factor

$$
F=1+\frac{4 \pi G \rho}{\Omega^{2}}=1+6.7\left(\frac{\rho}{0.3 \mathrm{~g} \mathrm{~cm}^{-3}}\right)\left(\frac{r}{10^{10} \mathrm{~cm}}\right)^{3}
$$

is the enhancement in the vertical gravitational field due to the self-gravity of the ring. The negative radial stress $\Sigma_{x x}$ represents the hydrostatic pressure required to maintain the ring thickness. 
More generally, the shear stress is likely to be a complex function of the shear, the surface density, and perhaps also their histories. Analytic models for the constitutive relations of a dense ring of inelastically colliding particles are still in a primitive state, particularly when cohesive forces are present. On the other hand $N$-body simulations of ring dynamics, which will be an indispensable guide to the correct analytic theory, have so far been restricted to the Keplerian shear rate and do not include the possibility of cohesion.

Given our present ignorance, the only practical approach is to parametrize a fairly general set of constitutive relations, and investigate the behavior of the ring as a function of these parameters. We shall therefore assume that the shear stress and the radial stress are arbitrary nonlinear functions of the shear $s$ and the surface density $\mu$. We can deduce some plausible general properties of these stresses. The shear stress $\Sigma_{x y}(\mu, s)$ should vanish if the shear vanishes (if there is no shear then there is no stress). We also expect that $\Sigma_{x y}$ will become very large as the shear $s$ approaches $-2 \Omega$, since we have seen in $\S 4.1$ that circular orbits are unstable when $s<-2 \Omega$. It is also natural to assume that $\Sigma_{x y}$ has the same sign as the shear $s$; however, we do not assume that $\Sigma_{x y}$ is monotonic in $s$, and in subsequent sections will focus on instabilities that can arise when $\Sigma_{x y}$ is a decreasing function of $s$ in some interval. The radial stress $\Sigma_{x x}(\mu, s)$ will be negative if the ring behaves as a fluid that exerts a pressure force, but may become positive in regions of negligible shear when the ring material freezes.

\subsection{Linear stability}

As described above, we shall assume that the stresses are functions of the local shear and surface density,

$$
\Sigma_{x x}=f(\mu, s) \quad, \quad \Sigma_{x y}=g(\mu, s) .
$$

We then linearize the equations of motion (4) around a state in which the surface density is uniform, the unperturbed motion is Keplerian (eq. 6), and the stresses are constant. We assume that the perturbations vary as $\exp (i k x+\gamma t)$. We find

$$
\begin{aligned}
\gamma u_{1}-2 \Omega v_{1} & =\frac{i k}{\mu_{0}}\left(f_{\mu} \mu_{1}+i k f_{s} v_{1}\right), \\
\gamma v_{1}+\frac{1}{2} \Omega u_{1} & =\frac{i k}{\mu_{0}}\left(g_{\mu} \mu_{1}+i k g_{s} v_{1}\right), \\
\gamma \mu_{1}+i k \mu_{0} u_{1} & =0
\end{aligned}
$$


where $f_{s} \equiv(\partial f / \partial s)_{0}$, etc. The resulting dispersion relation is ${ }^{1}$

$$
\mu \gamma^{3}+k^{2} g_{s} \gamma^{2}+\left(\Omega^{2} \mu-k^{2} \mu f_{\mu}-\frac{1}{2} \Omega k^{2} f_{s}\right) \gamma+k^{4}\left(f_{s} g_{\mu}-f_{\mu} g_{s}\right)-2 \Omega k^{2} \mu g_{\mu}=0 .
$$

We can recover the viscous instability and overstability of $\S 3$ by neglecting the radial stress $(f=0)$ and assuming that the ring is a Newtonian fluid so that $g=\mu s \nu(\mu)$. Equation (15) then simplifies to

$$
\gamma^{3}+k^{2} \nu \gamma^{2}+\Omega^{2} \gamma+3 \Omega^{2} k^{2} \frac{d(\mu \nu)}{d \mu}=0
$$

If the viscosity is small, this equation has two possible solutions: either $\gamma=-3 k^{2} d(\mu \nu) / d \mu+$ $\mathrm{O}\left(\nu^{2}\right)$, corresponding to the viscous instability (eq. 2), or $\gamma= \pm i \Omega+k^{2}\left[\frac{3}{2} \mu(d \nu / d \mu)+\nu\right]$, corresponding to the viscous overstability (eq. 3 , with the bulk viscosity $\xi=-\frac{4}{3} \nu$ so that the radial stress vanishes).

Thus the viscous instability and overstability arise in the limit where the stresses $f, g \rightarrow$ 0 at fixed wavenumber $k$. In this paper, we will focus instead on the short-wavelength limit, in which $k \rightarrow \infty$ at fixed values of $f, g$. In this case the roots of the dispersion relation are given by

$$
\gamma=-\frac{k^{2} g_{s}}{\mu}+\mathrm{O}\left(k^{0}\right), \quad \gamma^{2}=k^{2}\left(f_{\mu}-f_{s} \frac{g_{\mu}}{g_{s}}\right)+\mathrm{O}\left(k^{0}\right) .
$$

The first of these is the largest in absolute value, and leads to a rapidly growing instability if and only if $g_{s}<0$. This instability does not arise in a Newtonian disk, in which $g=E s$ so that $g_{s}=E>0$.

Assuming that the stresses $f$ and $g$ are comparable in magnitude, and that $f_{s} \sim f / \Omega$ and $f_{\mu} \sim f / \mu$, with similar relations for $g$, the approximations that lead to the formula (17) are valid so long as $k^{2} g \gtrsim \Omega^{2} \mu$. If we generalize equation (11) to

$$
g(s, \mu)=F \frac{\mu^{3} \Omega^{2}}{\rho^{2}} w(s),
$$

where $w^{\prime}(s)$ is of order unity, then this condition becomes $\lambda \lesssim 2 \pi F^{1 / 2} h$, where $h=\mu / \rho$ is the effective thickness, and we have replaced the wavenumber $k$ by $2 \pi / \lambda$. For typical values of $F$ (eq. 12), the condition becomes $\lambda \lesssim 20 h$. We expect that our two-dimensional approximation remains valid so long as $\lambda \gtrsim h$, so there is roughly one decade in wavenumber in which the instability should be present whenever $w^{\prime}\left(s_{K}\right)<0$.

\footnotetext{
${ }^{1}$ This analysis neglects the effects of the ring self-gravity; these can easily be added to (15) and have no important effect on our conclusions.
} 
We have established that a strong short-wavelength instability is present in rings in which $\partial g / \partial s<0$, that is, in which the shear stress becomes larger in absolute value when the shear rate becomes smaller in absolute value. We now analyze the nonlinear behavior of this instability in a ring model that is simplified even further.

\section{A toy model}

In this section we investigate a simple fluid system that illustrates an instability similar to the one we derived in the preceding section. Consider a homogeneous two-dimensional system of viscous incompressible fluid with surface density $\mu$. The system is infinite in the $y$-direction, and all of its physical properties are assumed to be independent of $y$. The system is in shear flow in the $y$-direction, described by the velocity field $\mathbf{v}=(0, v(x, t))$. The system has periodic shearing boundary conditions in the $x$-direction; that is, $v(x+\Delta x, t)=$ $v(x, t)+s_{K} \Delta x$, where $\Delta x$ is the period in $x, s_{K}$ is the mean shear (i.e. the shear averaged over length scales much larger than $\Delta x$ ). The only non-trivial component of the Euler equation reads

$$
\frac{\partial v}{\partial t}=\frac{1}{\mu} \frac{\partial \sigma_{x y}}{\partial x}
$$

where $\sigma$ is the stress tensor.

We let $s(x, t) \equiv \partial v / \partial x$ denote the shear. We shall assume that the stress is a nonlinear function of the shear, $\sigma_{x y}=g(s)$. Differentiating equation (19) with respect to $x$, we obtain

$$
\frac{\partial s}{\partial t}=\frac{1}{\mu} \frac{\partial^{2}}{\partial x^{2}} g(s)
$$

The periodic boundary conditions require that

$$
\int_{x}^{x+\Delta x} s(x, t) d x=s_{K} \Delta x \quad \text { for all } x .
$$

Initially we assume that the system is in a state of uniform shear, $s(x, t)=s_{K}$. A linearized analysis of equation (20) reveals that small disturbances of the form $\exp (\gamma t+i k x)$ satisfy the dispersion relation

$$
\gamma=-\frac{k^{2} g^{\prime}\left(s_{K}\right)}{\mu}
$$

exactly the same as the largest root in equation (17). Thus we have reproduced the instability derived in $§ 4.4$ in a simpler system, in which we can more easily investigate the nonlinear evolution of the instability. 
The final state of an unstable system with $g^{\prime}\left(s_{K}\right)<0$ is straightforward to describe. First we define

$$
G(s) \equiv \int_{0}^{s} g\left(s^{\prime}\right) d s^{\prime}, \quad F(t) \equiv \int_{x}^{x+\Delta x} G(s(x, t)) d x .
$$

Using equation (20) and the boundary conditions, we have

$$
\frac{d F}{d t}=\int_{x}^{x+\Delta x} g(s) \frac{\partial s}{\partial t} d x=\frac{1}{\mu} \int_{x}^{x+\Delta x} g(s) \frac{\partial^{2}}{\partial x^{2}} g(s) d x=-\frac{1}{\mu} \int_{x}^{x+\Delta x}\left(\frac{\partial g}{\partial x}\right)^{2} d x \leq 0 .
$$

Since $F$ decreases with time, the system must approach a steady state as $t \rightarrow \infty$. The steady-state solutions of equation $(20)$ are those in which $g(s)$ is a linear function of $x$; the periodic boundary conditions require that the linear term vanishes, so $g(s)=$ constant in the steady state. However, the only solution of this form that satisfies the mean shear constraint (21) is $s=s_{K}$, which is unstable. The resolution of this apparent contradiction is that the final state of the unstable system is piecewise constant in the shear $s$, alternating between states $s_{-}$and $s_{+}$that satisfy $g\left(s_{-}\right)=g\left(s_{+}\right)=g\left(s_{K}\right)$. The fractions $f_{ \pm}$of the spatial interval $\Delta x$ that are occupied by each of these two phases are constrained by the relations

$$
f_{-}+f_{+}=1, \quad f_{-} s_{-}+f_{+} s_{+}=s_{K},
$$

where the second equality follows from equation (21). However, the equations provide no information on the spatial distribution of the two phases in the final state; for example, the characteristic domain size is unknown.

The evolution from a uniform but unstable initial state into two distinct phases is known as spinodal decomposition in studies of alloys and binary fluids; in this case the shear $s$ is the concentration of one of the two components, $g(s)$ is the chemical potential, the functional $F$ is the free energy of the system, and equation (21) corresponds to conservation of mass (Bray 1994). This conservation law places our model in the class of phase transitions with a conserved order parameter.

The function $g(s)$ must satisfy several constraints in any physically plausible system: (i) $g(s)$ is an odd function of $s$ (since the directions $+y$ and $-y$ are equivalent); (ii) $g(0)=0$ (since there is no stress if there is no shear); (iii) $g(s)$ has the same sign as $s$ (since the viscosity is positive). A functional form that is general enough for our purposes is

$$
g(s)=s\left(a s^{2}+b|s|+c\right)
$$

condition (iii) then requires that $a>0, c>0$, and $b>-(4 a c)^{1 / 2}$. 
With this form for $g(s)$, there is an unstable region $\left(g^{\prime}(s)<0\right)$ if and only if $b<$ $-(3 a c)^{1 / 2}$. When this condition is satisfied, all values of $|s|$ between $s_{a}>0$ and $s_{b}>0$ are unstable, where

$$
s_{a, b}=-\frac{b}{3 a} \pm \frac{\left(b^{2}-3 a c\right)^{1 / 2}}{3 a}
$$

The interval $s_{a}<|s|<s_{b}$ in which a uniform shear is unstable is called the spinodal interval (Figure 1).

There is also interesting behavior outside the spinodal interval. Suppose that the system is in uniform shear $s_{1}$ outside the spinodal interval, $s_{1}>s_{b}$. Suppose that there is another shear state outside the spinodal interval, $s_{2}<s_{a}$, such that $g\left(s_{1}\right)=g\left(s_{2}\right)$. Then if a small element $\delta x$ changes its shear state from $s_{1}$ to $s_{2}$, and the remaining fluid increases its shear rate from $s_{1}$ to $s_{1}+\delta s_{1}$ so as to satisfy the mean shear constraint (21), the resulting change in free energy is

$$
\delta F=\delta x\left[G\left(s_{2}\right)-G\left(s_{1}\right)-G^{\prime}\left(s_{1}\right)\left(s_{2}-s_{1}\right)\right]
$$

which is negative if

$$
G^{\prime}\left(s_{1}\right)=g\left(s_{1}\right)>\frac{G\left(s_{2}\right)-G\left(s_{1}\right)}{s_{2}-s_{1}} .
$$

Shear states in which this inequality is satisfied - the interval $s_{p}<s<s_{a}$ and $s_{b}<s<s_{q}$ in Figure 1 - are metastable, because the mixture of two distinct phases has a lower free energy.

Equation (20) is ill-posed, since the growth rate of the instability (22) becomes extremely large for short-wavelength disturbances. It is convenient, and physically plausible, to mitigate this violent instability by modifying equation (20) to

$$
\frac{\partial s}{\partial t}=\frac{1}{\mu} \frac{\partial^{2}}{\partial x^{2}} g(s)-\epsilon \frac{\partial^{4} s}{\partial x^{4}},
$$

where $\epsilon$ is a small positive constant related to the thickness of the interface. The additional term can be thought of as penalizing the growth of solutions with large gradients.

We now investigate the properties of solutions of equation (30).

It is simple to see that the mean shear is conserved,

$$
\frac{d}{d t} \int_{x}^{x+\Delta x} s(x, t) d x=0,
$$

which implies that if the mean shear condition (21) is satisfied initially then it is automatically satisfied for all time.

The dispersion relation for small disturbances is

$$
\gamma=-\frac{k^{2} g^{\prime}\left(s_{K}\right)}{\mu}-\epsilon k^{4}
$$



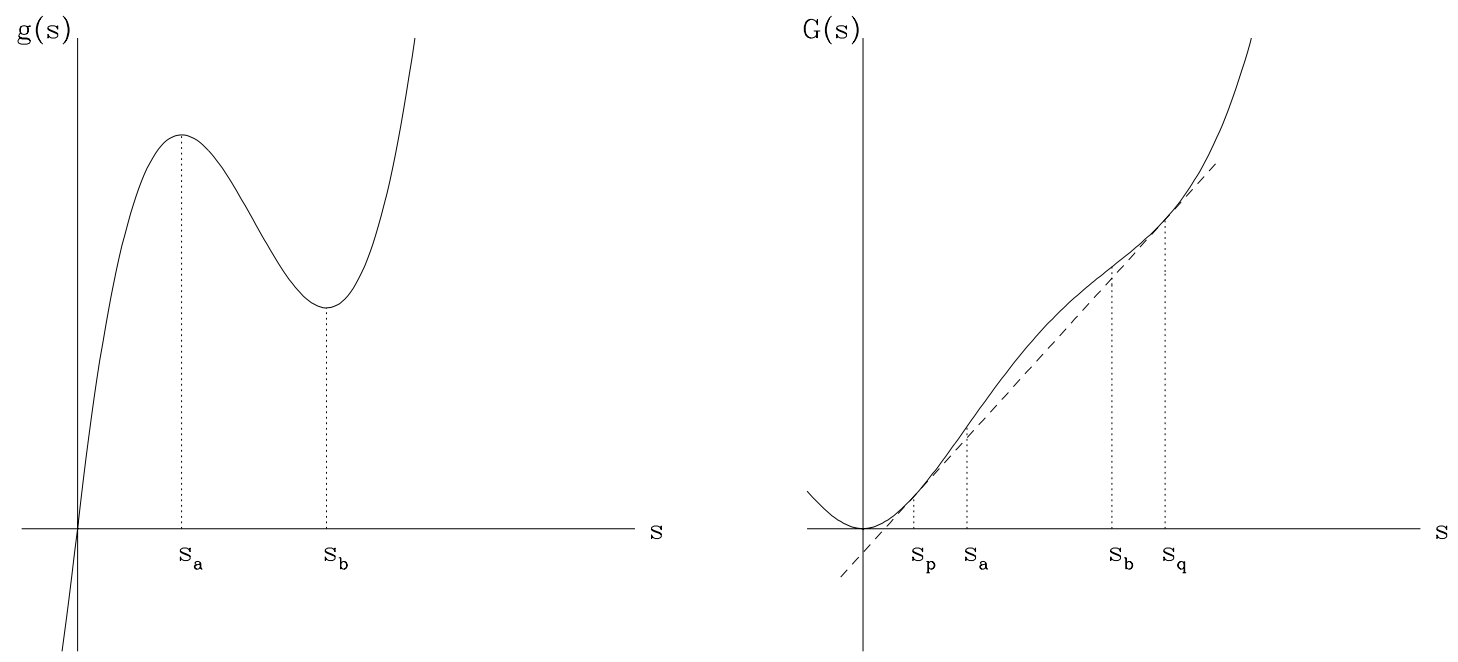

Fig. 1.- (left panel) A possible form for the shear stress $g(s)$ as a function of shear $s$, in the model of $\S 5$. Uniform shear is unstable in the interval $s_{a}<s<s_{b}$ (eq. 22). (right panel) The function $G(s)$ defined in equation (23), shown for the same parameters and on the same scale as the left panel. States in the interval $s_{p}<s<s_{a}$ and $s_{b}<s<s_{q}$ are metastable. The values $s_{p}$ and $s_{q}$ are determined by solving the equality corresponding to the inequality (29), along with the condition $g\left(s_{p}\right)=g\left(s_{q}\right)$. The solution of these two simultaneous equations is equivalent to finding the straight line tangent to $G(s)$ at two points. 
thus only wavenumbers that satisfy $k^{2}<-g^{\prime}\left(s_{K}\right) /(\epsilon \mu)$ are unstable; the most unstable wavenumber is given by $k_{\max }^{2}=-\frac{1}{2} g^{\prime}\left(s_{K}\right) /(\epsilon \mu)$.

Let us define

$$
F(t) \equiv \int_{x}^{x+\Delta x}\left[\frac{G(s(x, t))}{\mu}+\frac{1}{2} \epsilon\left(\frac{\partial s}{\partial x}\right)^{2}\right] d x,
$$

where $G(s)$ is defined in equation (23). It is straightforward to show for periodic boundary conditions that

$$
\frac{d F}{d t}=-\int_{x}^{x+\Delta x}\left(\frac{1}{\mu} \frac{\partial g}{\partial x}-\epsilon \frac{\partial^{3} s}{\partial x^{3}}\right)^{2} d x \leq 0 .
$$

Since $F$ decreases with time, the system must approach a steady state as $t \rightarrow \infty$.

The steady-state solutions of equation (30) are those in which $g(s) / \mu-\epsilon\left(d^{2} s / d x^{2}\right)$ is a linear function of $x$; the periodic boundary conditions require that the linear term vanishes, so the steady state solutions satisfy

$$
\frac{d^{2} s}{d x^{2}}-\frac{1}{\epsilon \mu} g(s)+p=0,
$$

where $p$ is a constant. This equation is equivalent to motion in the potential $-G(s) /(\epsilon \mu)+p s$, so there is an "energy" integral

$$
\frac{1}{2}\left(\frac{d s}{d x}\right)^{2}-\frac{1}{\epsilon \mu} G(s)+p s=q ;
$$

the properties of the steady-state solutions can thus be determined by examining the contours of the left-hand side of (36) in the phase plane with coordinates $(s, d s / d x)$.

We now introduce dimensionless variables,

$$
\xi \equiv\left(\frac{c}{\epsilon \mu}\right)^{1 / 2} x, \quad \tau \equiv \frac{c^{2}}{\epsilon \mu^{2}} t, \quad y \equiv\left(\frac{a}{c}\right)^{1 / 2} s ;
$$

recall that $a$ and $c$ are positive. Equation (30), with the stress-shear relation (26), becomes

$$
\frac{\partial y}{\partial \tau}=\frac{\partial^{2}}{\partial \xi^{2}}\left(y+B y|y|+y^{3}\right)-\frac{\partial^{4} y}{\partial \xi^{4}}, \quad B \equiv \frac{b}{(c a)^{1 / 2}} .
$$

The boundary conditions are periodic, with period $\Delta \xi \equiv(c / \epsilon \mu)^{1 / 2} \Delta x$. Equation (38) is the Cahn-Hilliard equation, which has been widely used to model pattern formation in phase transitions.

Consider small disturbances to uniform shear flow with shear $s_{K}$. The dispersion relation for disturbances of the form $\exp (\gamma \tau+i \kappa \xi)$ is

$$
\gamma=-\kappa^{2}\left(1+2 B\left|y_{K}\right|+3 y_{K}^{2}\right)-\kappa^{4},
$$


where $y_{K} \equiv(a / c)^{1 / 2} s_{K}$. There is instability if $\gamma>0$ for some $\kappa$, which occurs if

$$
B<-\frac{1+3 y_{K}^{2}}{2\left|y_{K}\right|} .
$$

If this instability is present, the stress as a function of shear $g(s)$ is triple-valued: there are three roots $s_{-}<s_{K}<s_{+}$to the equation $g(s)=g\left(s_{K}\right)$. The root $s_{K}$ has $g^{\prime}\left(s_{K}\right)<0$ and hence is unstable, while $g^{\prime}\left(s_{ \pm}\right)>0$ so the roots $s_{ \pm}$are stable.

Almost all of the steady-state solutions of equation (38) are bounded and periodic, and can be expressed analytically in terms of Jacobian elliptic functions Novick-Cohen \& Segel (1984). However, these are not the final state of the system: it turns out that all of the periodic solutions are unstable (Carr Gurtin \& Slemrod 1984). The only stable, stationary solution is the "kink" solution,

$$
y(x)= \pm \frac{1}{3} B \pm\left(\frac{1}{3} B^{2}-1\right)^{1 / 2} \tanh \left[\left(\frac{1}{3} B^{2}-1\right)^{1 / 2} \frac{x}{\sqrt{2}}\right],
$$

where the two \pm signs are independent and we require that $B<-3 / \sqrt{2}$. Of course, this solution does not satisfy our periodic boundary conditions.

Numerical integration of the partial differential equation (38) for a unstable initial state shows that the system evolves to a state in which the shear is almost always nearly equal to either $s_{-}$or $s_{+}$, just like the solutions of the simpler equation (20). Moreover, the interfaces between high- and low-shear domains gradually drift, so that high-shear and lowshear domains eventually coalesce. Once the distance between interfaces is large compared to unity, the shape of the shear curve in the transition region is given approximately by the kink solution (41). Because of the conservation law (31), the interfaces cannot move independently. Their interactions can be modeled by treating each interface as a particle that exerts forces on other particles (Kawasaki \& Ohta 1982; Majumdar \& Huse 1995). This process is known as coarsening or ripening. In an infinite system, the coarsening process continues indefinitely, although at a slower and slower rate as the domain sizes grow. Thus complete thermodynamic equilibrium is never achieved.

The coarsening process in the one-dimensional Cahn-Hilliard equation exhibits scaling behavior, that is, the characteristic distance between the interfaces grows indefinitely but the correlation function retains the same shape. However, in a planetary ring the coarsening will be halted when the distance between interfaces becomes comparable to the tidal width of $\S 4.2$. Thus our toy model leads naturally to the conclusion that the irregular structure in Saturn's rings should have a characteristic size comparable to the tidal width, which we estimated could be as large as $\sim 100 \mathrm{~km}$. 
Assuming that the characteristic width of the interface between solid and liquid phases is $L$, the parameter $\epsilon$ is of order $L^{2} \nu$ where $\nu$ is the kinematic viscosity. It is plausible that $L$ is comparable to the radius of large ring particles.

One interesting unresolved issue is whether our one-dimensional approximation is adequate. Coarsening in the Cahn-Hilliard equation is much faster for systems with more than one dimension, where the free energy associated with the interfaces or domain walls can be reduced by reducing their radius of curvature. Is the modest curvature of annuli in the B-ring sufficient to make this an important contributor to coarsening dynamics? A second unresolved issue is the importance of noise. The Cahn-Hilliard equation is a zero-temperature model; adding thermal noise converts the equation to a finite-temperature model. Is such a model more relevant for Saturn's B ring, and if so, what sets the effective temperature (impacts of small bodies? gravitational wakes from large ring particles?)? Are the fluctuations sufficient to trigger phase separation in the metastable region of the Cahn-Hilliard equation?

\section{Discussion}

We have suggested that the irregular structure in Saturn's B ring arises from the formation of solid ring-particle assemblies, which are limited in size by the competition between tidal forces and the tensile strength of these assemblies. We have shown that if the shear stress is a decreasing function of the shear, $\partial \Sigma_{x y} / \partial s<0$, then the ring is unstable at short wavelengths. Using a toy model of an incompressible, non-Newtonian fluid in shear flow, we have argued that this instability leads to the formation of domains in which the shear takes on one of two values; it is natural to identify one of these with the solid phase, and one with a liquid phase in which the (absolute value of the) shear rate is greater than Keplerian. The toy model is ill-posed at short wavelengths; if this is remedied by adding a term that can be associated with the energy of domain walls (the term proportional to $\epsilon$ in eq. 30), then the domains gradually coarsen, leading to structure on scales much larger than the original unstable wavelengths.

There are many shortcomings in this work. We have not derived a constitutive relation for a ring-particle fluid with cohesion, and thus we have not shown that the instability condition $\partial \Sigma_{x y} / \partial s<0$ is satisfied in rings with cohesive forces (although the simple kinetictheory model of Borderies, Goldreich \& Tremaine (1985) is marginally unstable; see eq. 11). We have not derived the statistical properties of the equilibrium ring structure, in which the growth of larger domains due to coarsening is balanced by their destruction from tidal forces. We have not discussed why the rigidly rotating solid regions should have different phase functions or albedo from the regions in which the particles are in differential rotation. 
We have not shown that solid annuli are dynamically stable. Finally, we have not established that ring particles stick to one another with the required cohesive strength; laboratory experiments suggest that a frost layer is needed but we do not know whether such layers are present in the $\mathrm{B}$ ring.

The most powerful tool we have to investigate at least some of these issues is $N$-body simulations of local ring dynamics. Such simulations have already provided considerable insight into the dynamics of rings composed of inelastically colliding particles (Wisdom \& Tremaine 1988; Richardson 1994; Mosqueira 1996; Salo, Schmidt \& Spahn 2001); what is required is to generalize them to include cohesion.

I thank David Huse and Roman Rafikov for helpful discussions, and Ignacio Mosqueira for detailed and thoughtful comments. This research was supported in part by NASA grant NAG5-10456.

\section{REFERENCES}

Araki, S., \& Tremaine, S. 1986, Icarus 90139

Asphaug, E., \& Benz, W. 1996, Icarus 121225

Borderies, N., Goldreich, P., \& Tremaine, S. 1985, Icarus 63406

Bray, A. J. 1994, Adv. Phys. 43357

Bridges, F. G., Supulver, K. D., Lin, D. N. C., Knight, R., \& Zafra, M. 1996, Icarus 123422

Carr, J., Gurtin, M. E., \& Slemrod, M. 1984, Arch. Rat. Mech. Anal. 86317

Chandrasekhar, S. 1961, Hydrodynamic and Hydromagnetic Stability (Oxford: Oxford University Press)

Cuzzi, J. N., Lissauer, J. J., Esposito, L. W., Holberg, J. B., Marouf, E. A., Tyler, G. L., \& Boischot, A. 1984, in Planetary Rings, ed. R. Greenberg and A. Brahic (Tucson: University of Arizona Press), 73

Daisaka, H., Tanaka, H., \& Ida, S. 2001, Icarus 154296

Durisen, R. H. 1995, Icarus 11566

Durisen, R. H., Cramer, N. L., Murphy, B. W., Cuzzi, J. N., Mullikin, T. L., \& Cederbloom, S. E. 1989, Icarus 80136 
Durisen, R. H., Bode, P. W., Cuzzi, J. N., Cederbloom, S. E., \& Murphy, B. W. 1992, Icarus 100364

Esposito, L. W., O'Callaghan, M., \& West, R. A. 1983, Icarus 56439

Fridman, A. M., Morozov, A. I., \& Polyachenko, V. L. 1984, Astrophys. Sp. Sci. 103137

Goldreich, P., \& Tremaine, S. 1978b, Icarus 34240

Hatzes, A. P., Bridges, F., Lin, D.N.C., and Sachtjen, S. 1991, Icarus 89113

Horn, L. J., \& Cuzzi, J. N. 1996, Icarus 119285

Kato, S. 1978, MNRAS 185629

Kawasaki, K., \& Ohta, T. 1982, Physica 116A 573

Lightman, A. P., \& Eardley, D. M. 1974, ApJ 187 L1

Lin, D.N.C., \& Bodenheimer, P. 1981, ApJ 248 L83

Lissauer, J. J. 1984, Icarus 5763

Lukkari, J. 1981, Nature 292433

Majumdar, S. N., \& Huse, D. A. 1995, Phys. Rev. E 270

Mosqueira, I. 1996, Icarus 122128

Mosqueira, I., \& Estrada, P. R. 2002, Icarus 158545

Novick-Cohen, A., \& Segal, L. A. 1984, Physica D 10277

Richardson, D. C. 1994, MNRAS 269493

Salo, H., Schmidt, J., \& Spahn, F. 2001, Icarus 153295

Schmidt, U., \& Tscharnuter, W. M. 1995, Icarus 115304

Schmidt, U., \& Tscharnuter, W. M. 1999, Icarus 138173

Schulson, E. M. 1999, JOM 5121

Showalter, M. R., \& Nicholson, P. D. 1990, Icarus 87285

Supulver, K. D., Bridges, F. G., Tiscareno, S., Lievore, J., \& Lin, D.N.C. 1997, Icarus 129 539 
Ward, W. R. 1981, Geophys. Res. Lett. 8641

Wisdom, J., \& Tremaine, S. 1988, Astron. J. 95925

Zebker, H. A., Marouf, E. A., \& Tyler, G. L. 1985, Icarus 64531 